\title{
On-Chip Terahertz-Frequency Measurements of Liquids
}

\author{
Matthew Swithenbank, ${ }^{\dagger}$ Andrew D. Burnett, ${ }^{\dagger}$ Christopher Russell, ${ }^{\dagger}$ Lianhe H. Li, $^{\dagger}$ \\ Alexander Giles Davies, ${ }^{\dagger}$ Edmund H. Linfield, ${ }^{\dagger}$ John E. Cunningham, ${ }^{\dagger}$ and Christopher D. Wood ${ }^{*}{ }^{\dagger}$ \\ ${ }^{\dagger}$ School of Electronic and Electrical Engineering, and ${ }^{\ddagger}$ School of Chemistry, University of Leeds, Leeds LS2 9JT, U.K.
}

Supporting Information

ABSTRACT: Terahertz-frequency-range measurements can offer potential insight into the picosecond dynamics, and therefore function, of many chemical systems. There is a need to develop technologies capable of performing such measurements in aqueous and polar environments, particularly when it is necessary to maintain the full functionality of biological samples. In this study, we present a proof-of-concept technology comprising an on-chip planar Goubau line, integrated with a microfluidic channel, which is capable of low-loss, terahertz-frequency-range spectroscopic measurements of liquids. We also introduce a mathematical model that accounts for changes in the electric field distribution around the waveguide, allowing accurate, frequencydependent liquid parameters to be extracted. We demonstrate the sensitivity of this technique by measuring a homologous alcohol series across the $0.1-0.8 \mathrm{THz}$ frequency range.
$\mathrm{T}$ he picosecond time scale (terahertz-frequency range) vibrational dynamics of many molecular systems are key to their chemical functionality ${ }^{1,2}$ and can be uniquely associated with structure. ${ }^{3,4}$ Contributions to short-range atomic and molecular dynamics in the infrared-frequency range have been thoroughly investigated; ${ }^{5,6}$ however, long-range and intermolecular vibrational modes occurring on picosecond time scales (corresponding to terahertz frequencies), that relate to molecular conformation, ${ }^{7}$ reaction dynamics, ${ }^{8}$ hydration, ${ }^{9}$ and biological function, ${ }^{1}$ are particularly difficult to probe using current spectroscopic techniques and are therefore poorly understood. In recent years, terahertz-frequency time-domain spectroscopy (THz-TDS) has been developed to investigate the picosecond characteristics of a wide range of materials, including explosives ${ }^{10}$ and drugs of abuse, ${ }^{11}$ and more recently, to interrogate the far-infrared vibrational modes of biological systems. ${ }^{1}$ Although significant information has been obtained from dry, pelletized samples, these often do not represent physically relevant environments; for example, measurements in aqueous environments are typically required in order to maintain full functionality of biological samples. ${ }^{1}$ However, the strong absorption of $\mathrm{THz}$-frequency signals in aqueous solutions necessitates that the interaction volume between the sample and probing radiation is severely restricted to avoid large attenuation of the $\mathrm{THz}$ signal. Where liquid samples are contained within a flow cell, ${ }^{12}$ the narrow separation between the cell windows required for transmission through these highly attenuating samples limits the measured frequency resolution (owing to the restricted time-domain duration) and complicates the interpretation of data (owing to the introduction of etalons). ${ }^{1}$ Windowless systems in which a free-flowing liquid sample is supported between two wires have been demon- strated, but the optical influence of the biconcave liquid cross section requires precise calibration. ${ }^{13,14}$ Alternatively, attenuated total reflection ${ }^{15}$ (ATR) and $\mathrm{THz}$ reflection spectroscopy $^{16}$ can be used to extract sample parameters from $\mathrm{THz}$ waves reflected off a window-liquid interface by modeling the interaction between the terahertz-frequency evanescent field and the analyte, thereby avoiding the need for propagation through the sample. ATR systems have been used to measure the absorption spectra of both solids and liquids, ${ }^{17,18}$ and imaging techniques have been used to observe protein crystallization. ${ }^{19}$ It is also possible to bond microfluidic channels directly to an ATR-crystal surface in order to measure liquid flows, ${ }^{20}$ and analyze overlaid samples. ${ }^{21}$ In summary, although methods for accurate, reliable, and repeatable terahertz-frequency measurements of compact liquid systems exist, they are in their infancy, and further advances are required to enable high-frequency-resolution measurements of strongly attenuating samples.

The integration of microfluidic systems with on-chip terahertz waveguides offers a compelling potential solution, since picosecond pulses confined to a planar transmission line can interact with nano- or microliter sample volumes over relatively long (millimeter) distances, with significantly reduced attenuation compared to free-space propagation. ${ }^{22}$ In fluidic channels with dimensions of a few hundred micrometers or less, interfacial forces, surface tension, and capillary effects dominate over gravitational forces to produce laminar flows. ${ }^{23}$ These controlled, predictable flows aid the design of micro-

Received: April 3, 2017

Accepted: July 6, 2017

Published: July 6, 2017 
mixers $^{24}$ and microreactors ${ }^{25}$ in which microliter or nanoliter volumes of scarce or costly samples can be controllably manipulated.

Planar waveguides have been widely investigated for the transmission of on-chip THz-frequency-range signals, ${ }^{26,27}$ but the inherent design restrictions of coplanar and microstrip geometries (the most common planar transmission line structures) limit their integration with microfluidic circuits. For example, in microstrip and coplanar transmission lines designed for operation at $\mathrm{THz}$ frequencies, the narrow (approximately tens of micrometers or smaller) separation required between the signal conductor and ground plane limits the extent of evanescent electric field, and therefore the interaction with overlaid samples. The planar Goubau line (PGL) is a transmission line that comprises a single-wire conductor, patterned on a low-permittivity substrate. THzfrequency signals guided along PGLs exhibit evanescent electric fields with a frequency-dependent extent of order $100 \mu \mathrm{m}$, significantly greater than that of equivalent coplanar or microstrip devices. For this reason, the PGL is well-suited to spectroscopic measurements of overlaid materials, owing to the increased interaction volume between the evanescent field and overlaid samples. We have previously used such devices to perform spectroscopy of powdered solids, in which we measured phonon-like, sharp vibrational modes. ${ }^{28}$ However, when using these systems for measurement of liquids, the integration of fluid-guiding channels can introduce Fresnel reflections from the interfaces between low- and highpermittivity regions along the PGL length, as illustrated in Figure $1 .{ }^{29}$ Here we demonstrate that the sources of these reflections can be removed by orienting the microfludic channel and waveguide such that the liquid under test covers the entirety of the PGL sensing region. ${ }^{29,30}$ However, even when such measures are applied, the extraction of quantitative information from measurements using planar transmission lines has previously been extremely problematic. This is primarily owing to a nonlinear modification of the waveguide properties (specifically, of the propagation modes) which occurs in the presence of an overlaid liquid; any quantitative analysis must therefore be able to correct for changes in the measured signal arising from the disruption or modification of these modes, allowing changes in signal caused by direct interaction with vibrational modes in an overlaid material to be assessed accurately. In this paper we demonstrate a numerical waveguide model that allows us to calculate accurate material parameters from proof-of-concept spectroscopic measurements of liquids, confined to microfluidic channels and integrated with our PGL devices. We determine the sensitivity of this technique by first measuring a homologous alcohol series in which controllable changes to molecular composition can be studied. We then use this technique to demonstrate the sensitivity of our device to water content by measuring a range of aqueous solutions (water/alcohol mixtures).

\section{EXPERIMENTAL SECTION}

The device used in this work comprised a $1 \mathrm{~mm}$ long PGL transmission line patterned over two photoconductive (PC) low-temperature-grown (LT) GaAs switches used for the generation and detection of picosecond $(\mathrm{THz})$ pulses. The PC switches were fabricated using techniques described elsewhere, ${ }^{31}$ and both the PGL and switches were defined on a $100 \mu \mathrm{m}$ thick double-side polished fused-quartz substrate, allowing through-substrate illumination of the PC switches. A

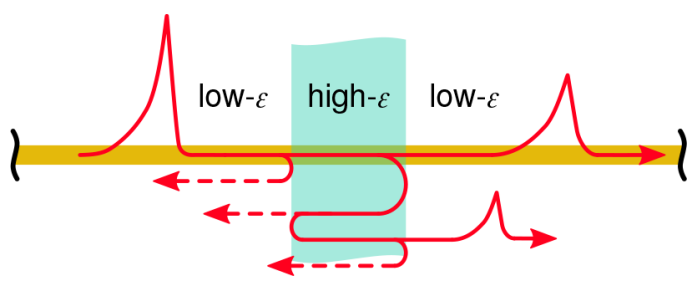

Figure 1. An electromagnetic pulse propagating along a conductor is partially reflected at the interfaces between regions of different permittivity, such as those created by an overlaid analyte or microfluidic channel.

$6 \mu \mathrm{m}$ thick layer of benzocyclobutene (BCB) was then deposited onto the PGL by spin-coating to form an electrically isolating layer.

A $100 \mu \mathrm{m}$ deep fluidic channel was defined by casting degassed poly(dimethylsiloxane) (PDMS, prepared in a 1:10 curing agent-to-base ratio) onto an SU-8 mold followed by curing at $80{ }^{\circ} \mathrm{C}$ for $1 \mathrm{~h}$. The cured PDMS was peeled off the mold, and a $0.75 \mathrm{~mm}$ diameter biopsy punch was used to create fluidic inlet and outlet holes at the ends of the PDMS channel. The structure was then bonded to the $\mathrm{BCB}$ surface using an irreversible amine-epoxy bonding technique, ${ }^{32}$ in which the two faces to be bonded were exposed to a $50 \mathrm{~W} \mathrm{O}_{2}$ plasma for $1 \mathrm{~min}$, forming hydroxyl groups on the surface. A $2 \%(\mathrm{v} / \mathrm{v})$ solution of 3-aminopropyltriethoxysilane and deionized (DI)$\mathrm{H}_{2} \mathrm{O}$ was poured over the BCB-coated device, and a $2 \%(\mathrm{v} / \mathrm{v})$ solution of 3-glycidoxypropyltriethoxysilane and propan-2-ol was poured over the PDMS channel. The devices were left in their respective solutions for $20 \mathrm{~min}$, during which the aminosilane and epoxysilane were anchored to the $\mathrm{O}_{2}$-treated surfaces. The two functionalized surfaces were brought into contact and left for $1 \mathrm{~h}$, until a bond was formed between the $\mathrm{BCB}$ layer and the PDMS. Fluid flow through the channel was actuated using a computer-controlled syringe pump (AL-1000, World Precision Instruments).

Illustrations of the completed device are shown in Figure 2, parts $a$ and $b$, in which the location of the photoconductive switches and the alignment of the microfluidic channel with respect to the transmission line are indicated. To determine the permittivity of an overlaid sample, the analysis technique introduced requires accurate measurements of each component of the device structure. The thickness of each layer in the device cross section was therefore measured during fabrication using a noncontact gauge, or a calibrated surface profiler as appropriate. A scale drawing of the resulting layered structure is shown in Figure 2c.

The completed device was mounted on a printed circuit board, fitted with SMA connectors to interface with further instrumentation, and then positioned in a TDS system, as shown in Figure $2 \mathrm{~d}$. To produce $\mathrm{THz}$ signals in the PGL, optical pulses from a Ti:sapphire laser (Tsunami, SpectraPhysics; $100 \mathrm{fs}$ duration, $800 \mathrm{~nm}$ center wavelength, $80 \mathrm{MHz}$ repetition rate, $10 \mathrm{~mW}$ average power) were focused through the substrate onto the generation switch (PC 1, Figure 2b), to which a $20 \mathrm{~V}$ bias was applied. An optically delayed, mechanically chopped $(1.8 \mathrm{kHz})$ beam was focused onto the detection switch, which allowed the resultant picosecond pulse to be measured coherently using lock-in detection. The timedomain response was recorded by measuring the transient photocurrent of the detection switch as the arrival time of the optical probe pulse was varied. ${ }^{33}$ 


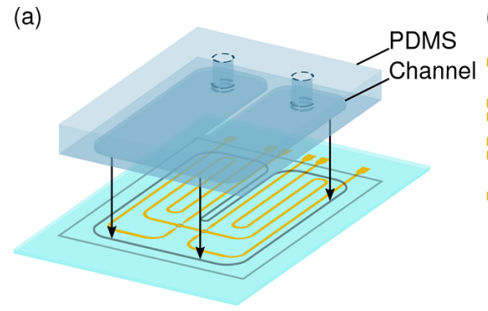

(b)

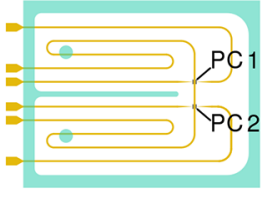
Delay stage (c)

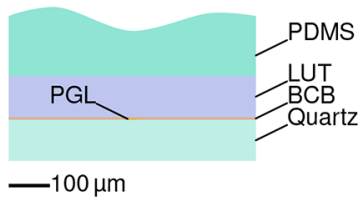

(d)

Figure 2. (a) Three-dimensional and (b) plan-view schematic illustration of the integrated PGL device with an overlaid microfluidic channel. The locations of the generation and detection switches (PC 1 and PC 2, respectively) are indicated, and the long parasitic arms outside this region are designed to delay the arrival time of unwanted system reflections. The positions of the inlet and outlet ports are indicated by the blue circles. (c) A cross section of the device comprising the quartz substrate, BCB insulation layer, LUT, and the PDMS channel boundary, drawn to scale. Note: the PDMS layer was approximately $4 \mathrm{~mm}$ thick, and so the top surface is not shown here. (d) Schematic arrangement of optical components used for the on-chip THz-TDS measurement system.

Time-domain measurements were first recorded for a reference (defined as the "empty" air-filled channel), followed by the sample, for which the device was first flushed with $3 \mathrm{~mL}$ of propan-2-ol, prior to filling with the liquid under test (LUT) at a flow rate of $5 \mu \mathrm{L} \mathrm{s}^{-1}$. Figure 3 shows the mean of five timedomain traces recorded for each of air, propan-1-ol, and methanol, for which the standard deviation was $<1 \%$, and therefore negligible. The peak center of the air reference was defined as 0 ps, from which the relative attenuation and propagation delay introduced by the sample loads is clear.

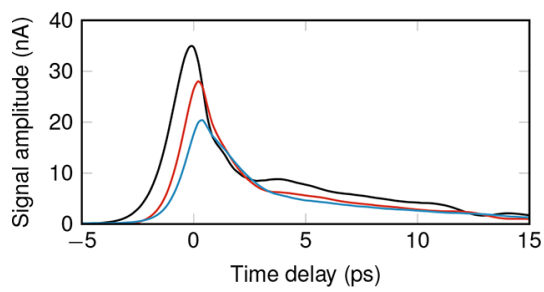

Figure 3. Pulses measured after propagation through a device when loaded with an air reference (black) and with propan-1-ol (red) and methanol (blue). A 200 ps time window was recorded, but only the first 20 ps are shown here for clarity.

\section{RESULTS AND DISCUSSION}

The sensitivity and accuracy of our measurement system were verified by using it to first extract the complex permittivity of a well-studied homologous alcohol series (methanol, ethanol, propan-1-ol), in order to allow quantitative comparison with published data. We then extended the alcohol series to include butan-1-ol, hexan-1-ol, and octan-1-ol, and finally demonstrated the limits of this particular device geometry through measurement of some highly attenuating propan-2-ol/DI- $\mathrm{H}_{2} \mathrm{O}$ mixtures. Here, the complex permittivity $\tilde{\varepsilon}_{\text {lut }}=\varepsilon_{\text {lut }}^{\prime}-\mathrm{i} \varepsilon_{\text {lut }}^{\prime \prime}$ of the given samples is presented (where $\varepsilon^{\prime}$ and $\varepsilon^{\prime \prime}$ represent the real and imaginary components, respectively), from which the complex refractive index can be calculated using $\tilde{n}=\sqrt{ } \tilde{\varepsilon}$, and the absorption coefficient from

$$
\alpha=\frac{2 \omega n^{\prime \prime}}{c}
$$

where $\omega$ is the angular frequency and $c$ is the speed of light in a vacuum.

To calculate accurately the frequency-dependent complex permittivity of a sample from time-domain measurements such as those shown in Figure 3, an understanding of the interaction between the THz electric field and the LUT is required. While the distribution of the electric and magnetic fields around traditional planar transmission line geometries, such as coplanar or microstrip, can be represented accurately using analytical models, ${ }^{34}$ similar models do not exist for the PGL. Previously, the real part of permittivity of a sample overlaying a PGL has been estimated by assuming a circular electric field distribution about the signal conductor. ${ }^{28}$ However, finite element simulations show that the electric field is in fact (as expected for any wave-guiding system) strongly dependent on the complex permittivity of the surrounding materials. For example, the evanescent electric field demonstrates greater substrate confinement for a PGL formed on a higher-permittivity material, with a correspondingly reduced evanescent field extent in the typically lower-permittivity superstrate. ${ }^{35}$ Indeed, there is a constant interplay between the relative values for permittivity of the substrate and any overlaid material; thus, a change in the overlaid material effectively alters the measurement system itself, which has previously prohibited detailed quantitative spectroscopic analysis using such systems. To improve the accuracy of the sample parameters extracted, it is therefore necessary to consider both the THz-frequency and complex permittivity dependence of the electric field distribution.

A finite element simulation of the device structure was performed in Ansys HFSS, ${ }^{36}$ using terahertz-frequency material properties of PDMS, ${ }^{37}$ quartz, ${ }^{38}$ and $\mathrm{BCB}^{39}$ taken from literature and the geometric dimensions of the fabricated 
device. The propagation constant, $\gamma=\zeta+\mathrm{i} \beta$, describes the change in amplitude and phase of a traveling wave, where $\zeta$ and $\beta$ are the per-unit-length attenuation and phase coefficients, respectively. When the channel is filled with an LUT during measurements, $\gamma$ rises, owing to an associated net increase in the complex permittivity of the materials with which the $\mathrm{THz}$ frequency evanescent field interacts. Using HFSS, the propagation constant of the unloaded Goubau mode was calculated between 0.1 and $1 \mathrm{THz}$. To allow calculation of the complex permittivity of an unknown LUT, $\gamma$ was simulated for a matrix of proxy LUTs with a constant (frequencyindependent) permittivity between $\tilde{\varepsilon}_{\text {lut }}=1-\mathrm{i} 0$ and $\tilde{\varepsilon}_{\text {lut }}=5-\mathrm{i} 6$, i.e.:

$$
\left(\begin{array}{cccc}
1-\mathrm{i} 0 & 1-\mathrm{i} 0.2 & \cdots & 1-\mathrm{i} 6 \\
1.5-\mathrm{i} 0 & 1.5-\mathrm{i} 0.2 & \cdots & 1.5-\mathrm{i} 6 \\
\vdots & \vdots & \ddots & \vdots \\
5-\mathrm{i} 0 & 5-\mathrm{i} 0.2 & \cdots & 5-\mathrm{i} 6
\end{array}\right)
$$

The frequency-dependent propagation constant calculated for each proxy sample is related to the complex effective permittivity of the modes by ${ }^{40}$

$$
\tilde{\varepsilon}_{\text {eff }}=\left(\mathrm{i} \frac{\gamma c}{\omega}\right)^{2}
$$

To demonstrate how these simulation results can be used to interpret data measured during an on-chip THz-TDS measurement, we first consider the Fourier transform of the timedomain data shown in Figure 3. In a typical free-space $\mathrm{THz}$ TDS measurement, the frequency-dependent phase shift, $\varphi$, is used to determine the real permittivity of a sample by adding the difference represented by $\varphi$, to the real permittivity of an air reference (i.e., 1). Here, rather than dry air, the reference measurement is of a device in which the microfluidic channel is filled with air, and therefore the simulated propagation constant of the Goubau mode in an air-filled reference device, $\gamma_{r}$, is used. The phase shift per unit length, $\Delta \beta$, introduced by the sample, was calculated by subtracting the complex phase angle of the LUT measurement from that of the reference, to determine $\varphi$, which was then divided by the $1 \mathrm{~mm}$ length of the transmission line to obtain $\Delta \beta$. The phase coefficient of the Goubau mode when the device was loaded with a sample, $\beta_{\text {s }}$, was found by adding the measured $\Delta \beta$ to the simulated phase coefficient when the device was filled with air, $\beta_{\mathrm{r}}$, such that $\beta_{\mathrm{s}}=\beta_{\mathrm{r}}+\Delta \beta$.

The attenuation coefficient, $\zeta$, is related to the signal amplitude by the Beer-Lambert law ${ }^{41}$

$$
\frac{A_{0}}{A_{l}}=\mathrm{e}^{\zeta l}
$$

where $A_{0}$ is the amplitude of the field generated at PC 1 and $A_{l}$ is the amplitude of the field after propagation over the distance $l$, measured at PC 2. Although the experimental configuration does not enable simultaneous measurement of $A_{0}$ and $A_{l}$, the generated field can be assumed to be constant between the reference and sample measurements. Therefore, eq 4 can be rearranged to remove $A_{0}$ :

$$
\zeta_{\mathrm{s}}=\frac{1}{l} \ln \left(\frac{A_{l, \mathrm{r}}}{A_{l, \mathrm{~s}}}\right)+\zeta_{\mathrm{r}}
$$

where $\zeta_{\mathrm{s}}$ and $\zeta_{\mathrm{r}}$ are the sample-loaded and reference attenuation coefficients of the Goubau mode and $A_{l, x}$ is the amplitude of the

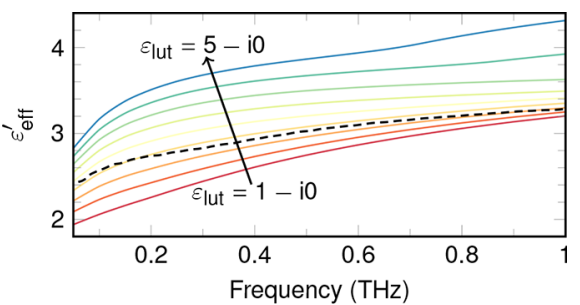

Figure 4. Simulated effective real permittivity of the Goubau mode when the complex permittivity of the proxy sample was varied from $\varepsilon_{\text {lut }}=1-\mathrm{i} 0$ to $\varepsilon_{\text {lut }}=5-\mathrm{i} 0$ (solid) in increments of $0.5-\mathrm{i} 0$, and the measured $\varepsilon_{\text {eff }}^{\prime}$ of the Goubau mode when the device was loaded with propan-1-ol (dashed).

reference $(x=\mathrm{r})$ and sample-loaded $(x=\mathrm{s})$ field measured at PC 2, as indicated by the subscript. The total propagation constant of the sample-loaded Goubau mode is therefore $\gamma_{\mathrm{s}}=\beta_{\mathrm{s}}$ $+\mathrm{i} \zeta_{s}$, from which the effective permittivity of the mode can be determined using eq 3 . In this work, our method is applied to the measurement of liquid analytes; however, the same approach could be used in the analysis of overlaid solid samples. Given that the permittivity is determined independently at each frequency point, we anticipate that the technique could be used to accurately resolve resonant spectral features in the same manner.

In Figure 4, the measured effective permittivity of the mode when the device was loaded with propan-1-ol is plotted against the simulated effective permittivity when loaded with proxy samples. The real permittivity of propan-1-ol is not constant across the frequency range considered here; hence, the measured $\varepsilon_{\text {eff }}^{\prime}$ does not align with any one trace. From investigation of the simulation results, the real component of the mode permittivity was found to be independent of the imaginary component. Therefore, the dimensionality of the problem was reduced by first fitting $\varepsilon_{\text {lut }}^{\prime}$ at each frequency point in turn, with a two-dimensional cubic interpolation algorithm by assuming $\varepsilon_{\text {lut }}^{\prime \prime}=0$ at all frequencies (i.e., interpolation along the first column of the proxy LUT matrix). ${ }^{42}$ Similar interpolation methods have been used in the analysis of $\mathrm{THz}$ TDS systems with complex propagation models. ${ }^{43,44}$ In contrast, the imaginary component was found to be strongly dependent on the real permittivity, owing to the substantial changes in electric field distribution that occur as a function of $\varepsilon_{\text {lut. }}^{\prime}$ Therefore, the imaginary component was calculated by interpolation based on the real component that had been found (e.g., if a real component $\varepsilon_{\text {lut }}^{\prime}=1.5$ had been identified, then the imaginary part would be found by interpolation along the second row of the proxy LUT matrix).

In Figure 5, our measured values of complex permittivity for methanol, ethanol, and propan-1-ol are compared to data presented by Barthel et al., ${ }^{45}$ in which samples were measured at frequencies accessible by rectangular waveguide bands, Kindt and Schmuttenmaer, ${ }^{12}$ in which alcohols were measured using a free-space $\mathrm{THz}$ transmission system, incorporating a flowcell fabricated from high-density polyethylene and high-resistivity silicon, Yomogida et al., ${ }^{46}$ who used a free-space THz-TDS system to measure liquids contained between polypropylene windows, Hirori et al., ${ }^{47}$ who measured methanol using an ATR system, and Møller et al., ${ }^{16}$ who employed a reflection spectrometer to measure ethanol. Considering the range of experimental configurations, and analysis methods implemented in the literature, it is encouraging to observe a relatively strong agreement between the sample permittivity 

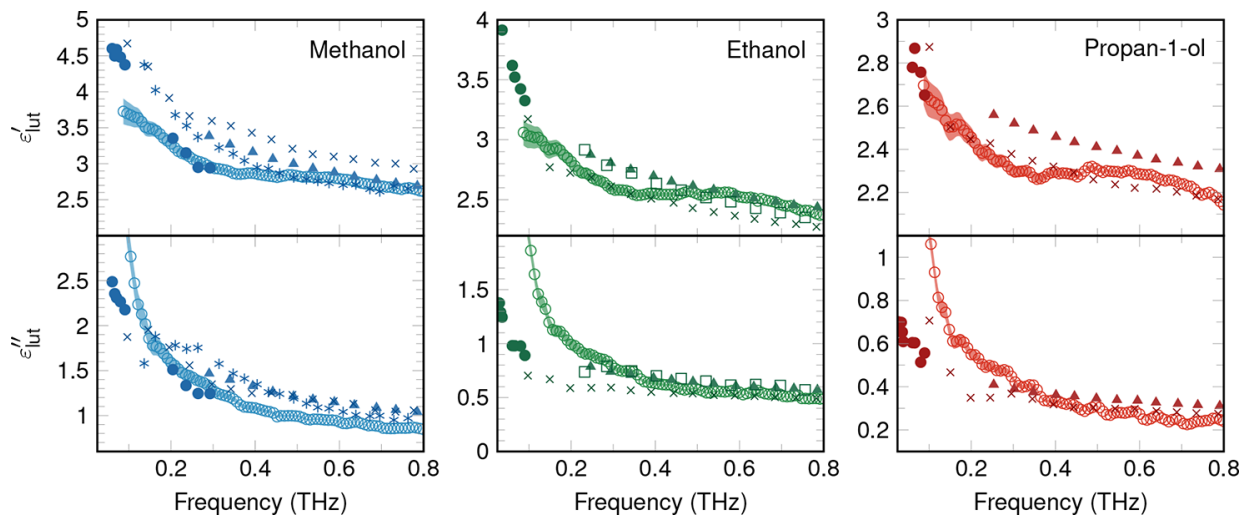

Figure 5. Complex permittivity of methanol, ethanol, and propan-1-ol. For each sample, the real and imaginary permittivity measured in this work ( deg) is plotted along with the permittivity measured by Barthel et al. (ref 45) (•), Kindt and Schmuttenmaer (ref 12) (X), and Yomogida et al. (ref 46) (A). Data recorded with ATR and reflection systems by Hirori et al. (ref 47) (*), and Møller et al. (ref 16) ( $\square$ ) are also included. The shaded regions represent the $\pm 10 \%$ channel-height model error of our measurements.

Table 1. Triple-Debye Model Dielectric Relaxation Parameters Calculated for Methanol, Ethanol, and Propan-1-ol, for Which the Values Given in Square Brackets and Parentheses Are Those Reported by Barthel et al. ${ }^{45}$ and Kindt and Schmuttenmaer, ${ }^{12}$ Respectively

\begin{tabular}{|c|c|c|c|c|c|c|c|}
\hline LUT & $\varepsilon_{\mathrm{DC}}$ & $\tau_{1}(\mathrm{ps})$ & $\varepsilon_{2}$ & $\tau_{2}(\mathrm{ps})$ & $\varepsilon_{3}$ & $\tau_{3}(\mathrm{ps})$ & $\varepsilon_{\infty}$ \\
\hline \multirow[t]{3}{*}{ methanol } & {$[32.63]$} & {$[51.5]$} & {$[5.91]$} & [7.09] & {$[4.9]$} & {$[1.12]$} & [2.79] \\
\hline & (32.63) & $(48)$ & $(5.35)$ & $(1.25)$ & $(3.37)$ & $(0.16)$ & $(2.10)$ \\
\hline & 32.63 & 51.01 & 5.52 & 1.84 & 2.85 & 0.16 & 2.37 \\
\hline \multirow[t]{3}{*}{ ethanol } & {$[24.35]$} & {$[163]$} & [4.49] & {$[8.97]$} & {$[3.82]$} & {$[1.81]$} & [2.69] \\
\hline & (24.35) & (161) & $(4.15)$ & $(3.3)$ & $(2.72)$ & $(0.22)$ & (1.93) \\
\hline & 24.35 & 161 & 4.45 & 4.16 & 3.34 & 0.69 & 2.24 \\
\hline \multirow[t]{3}{*}{ propan-1-ol } & {$[20.44]$} & [329] & {$[3.74]$} & {$[15.1]$} & {$[3.2]$} & {$[2.4]$} & {$[2.44]$} \\
\hline & (20.44) & $(316)$ & (3.43) & $(2.9)$ & $(2.37)$ & $(0.2)$ & (1.97) \\
\hline & 20.44 & 328 & 3.79 & 14.2 & 3.05 & 1.01 & 2.16 \\
\hline
\end{tabular}

values found using the differing techniques. Additionally, it is clear that the data recorded with the on-chip spectrometer developed in this work lie within the variance of the published data sets.

It is well-recognized that PDMS samples placed in contact with solvents (such as propan-1-ol) undergo swelling by up to $10 \%{ }^{48}$ Furthermore, since PDMS is an elastomer, excess pressure within the fluidic channel may lead to changes in the channel geometry and volume. The latter issue can be controlled by limiting the flow rate-a low flow rate of $5 \mu \mathrm{L} \mathrm{s}^{-1}$ was therefore chosen to minimize the induced pressure within the channel. Since our analysis technique relies on accurate measurement of the fluidic channel dimensions, we anticipate that the greatest source of error will therefore arise from solvent-induced swelling of the PDMS. To account for this possible change in device geometry, we repeated device simulations to estimate the maximum error introduced at the extremes of channel height, corresponding to $\pm 10 \%$ of our stated value of $100 \mu \mathrm{m}$. The corresponding difference in calculated permittivity is indicated by the shaded regions in Figure 5 .

In some of the previous works, the agreement between data sets was quantified by fitting a relaxation model to the complex permittivity of the analytes studied. ${ }^{12,45}$ The frequencydependent dielectric response of polar liquids can be described by a number of Debye-relaxation and harmonic-oscillator processes, which span several orders of magnitude in the frequency domain. ${ }^{46,49}$ For example, Kindt and Schmuttenmaer $^{12}$ fit their data to a triple-Debye relaxation model, which has been used to represent the intramolecular rotation, reorientation, and hydrogen bonding of protic solvents such as methanol, ethanol, and propan-1-ol. ${ }^{45,50}$ The frequency dependence of the Debye permittivity, $\tilde{\varepsilon}_{\mathrm{D}}$, is determined by the summation $^{12}$

$$
\tilde{\varepsilon}_{\mathrm{D}}=\varepsilon_{\infty}+\sum_{j=1}^{n} \frac{\varepsilon_{j}-\varepsilon_{j+1}}{1+\mathrm{i} \omega \tau_{j}}
$$

where $\varepsilon_{\infty}$ is the sample's high-frequency permittivity limit and $\varepsilon_{j}$ are the $n$ intermediate permittivity intervals, with the corresponding relaxation time constants $\tau_{j}$.

Here, a nonlinear least-squares algorithm, initialized using the parameters identified by Kindt and Schmuttenmaer, ${ }^{12}$ was used to fit $\varepsilon_{j}$ and $\tau_{j}$ to the data measured here between 100 and $800 \mathrm{GHz}$. In addition, the model was constrained at frequencies between 1 and $200 \mathrm{GHz}$ using the data presented by Barthel et al. ${ }^{45}$ In each fitting operation, $\varepsilon_{\mathrm{DC}}$ was fixed at the initialized value. $^{12}$ The parameters reported by Barthel et al., ${ }^{45}$ Kindt and Schmuttenmaer, ${ }^{12}$ and those found in this work, are presented in Table 1, and a good agreement is observed. The critical frequency, ${ }^{12} f_{c}=1 /\left(2 \pi \tau_{j}\right)$, of the fastest relaxation processes $\left(\tau_{3}\right)$ was outside the spectral range of the rectangular waveguide system, and also at the limit of the on-chip system in some cases (e.g., 0.2 ps $\equiv 795 \mathrm{GHz}$ ). Such processes are therefore difficult to accurately resolve with all systems, resulting in some disagreement in the parameters fitted to those terms. However, the parameters found to fit the on-chip spectrometer data, shown in Table 1, were typically between the values reported 


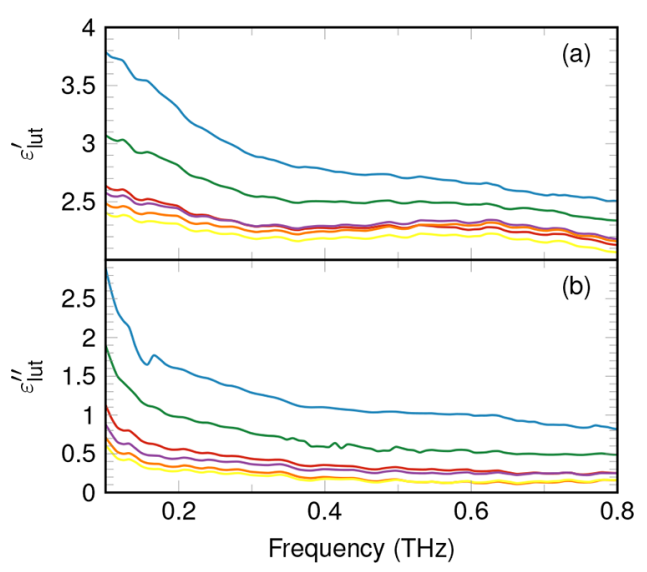

Figure 6. (a) Measured permittivity and (b) absorption coefficient of methanol (blue), ethanol (green), propan-1-ol (red), butan-1-ol (purple), hexan-1-ol (orange), and octan-1-ol (yellow).

for the lower-frequency rectangular waveguide system, ${ }^{45}$ and the higher-frequency free-space spectrometer. ${ }^{12}$

The higher-order alcohols butan-1-ol, hexan-1-ol, and octan1-ol were then measured with the on-chip spectrometer. Between measurements, the channel was flushed with propan2-ol and dry air, as before. Particular attention was paid when flushing the system of the higher-order alcohols as they were observed to leave a residue within the channel that required additional flushing of propan-2-ol to remove. The frequencydependent permittivities of each of the alcohols in Figure 6 were calculated, and a systematic decrease in both real and imaginary permittivity was observed as the alcohol series order was increased, demonstrating a sensitivity to molecular composition.

The ability to measure highly attenuating aqueous systems is critical for the study of samples in biologically relevant environments. Therefore, the limits of this particular geometry were next investigated by preparing propan-2-ol/DI- $\mathrm{H}_{2} \mathrm{O}$ mixtures in which the volume fraction of $\mathrm{DI}-\mathrm{H}_{2} \mathrm{O}$ was increased from $0 \%$ to $100 \%$ in increments of $10 \%$, which equate to the mole fractions, $\chi$, given in Table 2 . Using the results of our HFSS simulations, the frequency-dependent real and imaginary permittivities in Figure 7 were calculated. The results demonstrate clearly the sensitivity of this technique to changes in sample dilution for liquids with values of permittivity that vary between $\varepsilon_{\text {lut }}=2-\mathrm{i} 0.5$ and $\varepsilon_{\text {lut }}=5-\mathrm{i} 6$. However, the bandwidth that could be resolved for some of the more absorbing solutions (>70\% DI- $\mathrm{H}_{2} \mathrm{O}$ ) was severely restricted, and the significant attenuation of the $100 \%$ DI- $\mathrm{H}_{2} \mathrm{O}$ sample meant that the imaginary component of the permittivity was not successfully extracted. The bandwidth measurable with a particular device is restricted both by the attenuation of the electric field and by a preferential multimode propagation when the permittivity of materials in the proximity of the planar Goubau line is highly asymmetric. ${ }^{35}$ As with any THz-TDS system, there is an interplay between sensitivity and bandwidth. The measurable bandwidth of an on-chip microfluidic spectrometer can be increased by reducing the interaction volume between the propagating field and the analyte. For

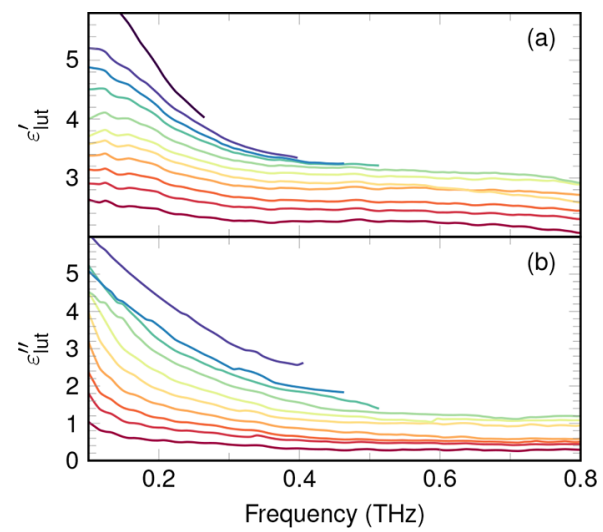

Figure 7. Measured (a) real and (b) imaginary permittivity of propan2 -ol/DI- $\mathrm{H}_{2} \mathrm{O}$ mixtures with volume fractions from $0 \%$ (red) to $100 \%$ (purple) DI- $\mathrm{H}_{2} \mathrm{O}$ in $10 \%$ increments. The significant attenuation of $100 \%$ DI- $\mathrm{H}_{2} \mathrm{O}$ sample meant that the loss could not be resolved.

example, Figure S-1 shows the time and frequency-domain response of the device discussed in this work (comprising a $100 \mu \mathrm{m}$ deep microfluidic channel), and the response of a device with a $20 \mu \mathrm{m}$ deep channel, when both devices were loaded with a DI- $\mathrm{H}_{2} \mathrm{O}$ sample. It can be seen that the reduction in channel height, corresponding to a decrease in the sample load, increased the dynamic range in the frequency domain between 0 and $800 \mathrm{GHz}$. Thus, the inherent flexibility in the design of an on-chip microfluidic spectrometer allows for optimization of the device to be most sensitive over a desired permittivity range.

\section{CONCLUSIONS}

A technology comprising a planar Goubau line THz-TDS system integrated with a microfluidic measurement system on a single chip has been introduced, and a method has been described in which a numerical model based on finite element simulations of the device structure when filled with proxy fixedpermittivity samples is used to extract the permittivity of an unknown liquid sample. A well-studied series of homologous alcohols was measured, and the permittivity measured for each sample was found to be in good agreement with available literature. The high-permittivity limits of the device geometry were investigated, and methods for changing the sensitive permittivity range were discussed. We anticipate that this technique can be used in future development and analysis of integrated terahertz measurement systems, particularly for the measurement of the high-frequency dynamics of aqueous- or solvent-based materials.

\section{ASSOCIATED CONTENT}

\section{Supporting Information}

The Supporting Information is available free of charge on the ACS Publications website at DOI: 10.1021/acs.analchem.7b01235.

Frequency spectrum of two devices loaded with $\mathrm{DI}-\mathrm{H}_{2} \mathrm{O}$, the first with a $100 \mu \mathrm{m}$ deep microfluidic channel, and the second with a $20 \mu \mathrm{m}$ deep channel, in which the

Table 2. DI- $\mathrm{H}_{2} \mathrm{O}$ Volume and Mole Fractions of the 11 Propan-2-ol/DI- $\mathrm{H}_{2} \mathrm{O}$ Solutions Measured

\begin{tabular}{|c|c|c|c|c|c|c|c|c|c|c|c|}
\hline vol fraction (\%) & 0 & 10 & 20 & 30 & 40 & 50 & 60 & 70 & 80 & 90 & 100 \\
\hline mole fraction $(\chi)$ & 0.00 & 0.32 & 0.51 & 0.64 & 0.74 & 0.81 & 0.86 & 0.91 & 0.94 & 0.97 & 1.00 \\
\hline
\end{tabular}


increase in measurable bandwidth resulting from the thinner channel is clearly observed (PDF)

\section{AUTHOR INFORMATION}

\section{Corresponding Author}

*E-mail: C.D.Wood@leeds.ac.uk. Phone: +44 01133438335. ORCID ${ }^{\circ}$

Matthew Swithenbank: 0000-0002-6146-1818

Notes

The authors declare no competing financial interest.

\section{ACKNOWLEDGMENTS}

The authors thank the Engineering and Physical Sciences Research Council (EP/L504993/1; EP/I026657/1) and the Defense Threat Reduction Agency (U.S.) (HDTRA1-14-C0013) and the Wellcome Trust $(201058 / \mathrm{Z} / 16 / \mathrm{Z})$. Support from the Royal Society and Wolfson Foundation is also acknowledged. The data associated with this paper are openly available from the University of Leeds data repository. ${ }^{51}$

\section{REFERENCES}

(1) Markelz, A. G. IEEE J. Sel. Top. Quantum Electron. 2008, 14, 180-190.

(2) Zhang, L.; Wang, L.; Kao, Y.-T.; Qiu, W.; Yang, Y.; Okobiah, O.; Zhong, D. Proc. Natl. Acad. Sci. U. S. A. 2007, 104, 18461-18466.

(3) Taday, P. F. Philos. Trans. R. Soc., A 2004, 362, 351-364.

(4) Fischer, B.; Hoffmann, M.; Helm, H.; Modjesch, G.; Jepsen, P. U. Semicond. Sci. Technol. 2005, 20, S246.

(5) Movasaghi, Z.; Rehman, S.; Rehman, I. U. Appl. Spectrosc. Rev. 2007, 42, 493-541.

(6) Movasaghi, Z.; Rehman, S.; ur Rehman, I. Appl. Spectrosc. Rev. 2008, 43, 134-179.

(7) Walther, M.; Plochocka, P.; Fischer, B.; Helm, H.; Uhd Jepsen, P. Biopolymers 2002, 67, 310-313.

(8) Hay, S.; Scrutton, N. S. Nat. Chem. 2012, 4, 161-168.

(9) Laurette, S.; Treizebre, A.; Affouard, F.; Bocquet, B. Appl. Phys. Lett. 2010, 97, 111904.

(10) Shen, Y. C.; Lo, T.; Taday, P. F.; Cole, B. E.; Tribe, W. R.; Kemp, M. C. Appl. Phys. Lett. 2005, 86, 241116.

(11) Davies, A. G.; Burnett, A. D.; Fan, W.; Linfield, E. H.; Cunningham, J. E. Mater. Today 2008, 11, 18-26.

(12) Kindt, J.; Schmuttenmaer, C. J. Phys. Chem. 1996, 100, 1037310379 .

(13) Tauber, M. J.; Mathies, R. A.; Chen, X.; Bradforth, S. E. Rev. Sci. Instrum. 2003, 74, 4958-4960.

(14) Wang, T.; Klarskov, P.; Jepsen, P. U. IEEE Trans. Terahertz Sci. Technol. 2014, 4, 425-431.

(15) Hirori, H.; Yamashita, K.; Nagai, M.; Tanaka, K. Jpn. J. Appl. Phys. 2004, 43, L1287-L1289.

(16) Møller, U.; Cooke, D. G.; Tanaka, K.; Jepsen, P. U. J. Opt. Soc. Am. B 2009, 26, A113-A125.

(17) Newnham, D. A.; Taday, P. F. Appl. Spectrosc. 2008, 62, 394398.

(18) Nagai, M.; Yada, H.; Arikawa, T.; Tanaka, K. Int. J. Infrared Millimeter Waves 2006, 27, 505-515.

(19) Chan, K. A.; Govada, L.; Bill, R. M.; Chayen, N. E.; Kazarian, S. G. Anal. Chem. 2009, 81, 3769-3775.

(20) Chan, K. A.; Gulati, S.; Edel, J. B.; de Mello, A. J.; Kazarian, S. G. Lab Chip 2009, 9, 2909-2913.

(21) Kazarian, S. G. Anal. Bioanal. Chem. 2007, 388, 529-532.

(22) Ohkubo, T.; Onuma, M.; Kitagawa, J.; Kadoya, Y. Appl. Phys. Lett. 2006, 88, 212511.

(23) Sackmann, E. K.; Fulton, A. L.; Beebe, D. J. Nature 2014, 507, $181-189$.

(24) Nguyen, N.-T.; Wu, Z. J. Micromech. Microeng. 2005, 15, R1R16.
(25) Kang, L.; Chung, B. G.; Langer, R.; Khademhosseini, A. Drug Discovery Today 2008, 13, 1-13.

(26) Frankel, M. Y.; Gupta, S.; Valdmanis, J. A.; Mourou, G. A. IEEE Trans. Microwave Theory Tech. 1991, 39, 910-916.

(27) Byrne, M.; Cunningham, J.; Tych, K.; Burnett, A.; Stringer, M.; Wood, C.; Dazhang, L.; Lachab, M.; Linfield, E.; Davies, A. Appl. Phys. Lett. 2008, 93, 182904.

(28) Russell, C.; Wood, C. D.; Burnett, A. D.; Li, L.; Linfield, E. H.; Davies, A. G.; Cunningham, J. E. Lab Chip 2013, 13, 4065-4070.

(29) Swithenbank, M.; Russell, C.; Burnett, A. D.; Li, L.; Linfield, E. H.; Davies, A. G.; Cunningham, J. E.; Wood, C. D. 40th International Conference on Infrared Millimeter and Terahertz Waves 2015, 40, 3-7.

(30) Russell, C.; Swithenbank, M.; Wood, C. D.; Burnett, A. D.; Li, L.; Linfield, E. H.; Davies, A. G.; Cunningham, J. E. IEEE Trans. Terahertz Sci. Technol. 2016, 6, 619-624.

(31) Cunningham, J.; Wood, C.; Davies, A.; Hunter, I.; Linfield, E.; Beere, H. Appl. Phys. Lett. 2005, 86, 213503.

(32) Tang, L.; Lee, N. Y. Lab Chip 2010, 10, 1274-1280.

(33) Hangyo, M.; Tani, M.; Nagashima, T. Int. J. Infrared Millimeter Waves 2005, 26, 1661-1690.

(34) Green, H. E. IEEE Trans. Microwave Theory Tech. 1965, 13, 676-692.

(35) Treizebré, A.; Bocquet, B.; Xu, Y.; Bosisio, R. G. Microw. Opt. Technol. Lett. 2008, 50, 2998-3001.

(36) HFSS v15, release 15.0.7; Ansys Inc.: Pittsburg, PA, 2014.

(37) Podzorov, A.; Gallot, G. Appl. Opt. 2008, 47, 3254-3257.

(38) Naftaly, M.; Miles, R. E. Proc. IEEE 2007, 95, 1658-1665.

(39) Perret, E.; Zerounian, N.; David, S.; Aniel, F. Microelectron. Eng. 2008, 85, 2276-2281.

(40) Schlereth, K.-H.; Tacke, M. IEEE J. Quantum Electron. 1990, 26, 627-630.

(41) Kasap, S. Principles of Electronic Materials and Devices, 3rd ed.; McGraw-Hill Education: Boston, MA, 2006.

(42) Withayachumnankul, W.; Naftaly, M. J. Infrared, Millimeter, Terahertz Waves 2014, 35, 610-637.

(43) Pupeza, I.; Wilk, R.; Koch, M. Opt. Express 2007, 15, 43354350.

(44) Baragwanath, A.; Swift, G.; Dai, D.; Gallant, A.; Chamberlain, J. J. Appl. Phys. 2010, 108, 013102.

(45) Barthel, J.; Bachhuber, K.; Buchner, R.; Hetzenauer, H. Chem. Phys. Lett. 1990, 165, 369-373.

(46) Yomogida, Y.; Sato, Y.; Nozaki, R.; Mishina, T.; Nakahara, J. J. Mol. Liq. 2010, 154, 31-35.

(47) Hirori, H.; Arikawa, T.; Nagai, M.; Ohtake, H.; Yoshida, M.; Tanaka, K. 2004 Joint 29 Int. Conf. on Infrared and Millimeter Waves and 12th Int. Conf. on Terahertz Electronics 2004, 251-252.

(48) Lee, J. N.; Park, C.; Whitesides, G. M. Anal. Chem. 2003, 75, $6544-6554$

(49) Fukasawa, T.; Sato, T.; Watanabe, J.; Hama, Y.; Kunz, W.; Buchner, R. Phys. Rev. Lett. 2005, 95, 197802.

(50) Barthel, J.; Buchner, R. Pure Appl. Chem. 1991, 63, 1473.

(51) Swithenbank, M.; Burnett, A.; Russell, C.; Li, L.; Davies, A. G.; Linfield, E.; Cunningham, J. E.; Wood, C. University of Leeds [Dataset], 2017. https://doi.org/10.5518/103. 